\title{
Long-read based assembly and annotation of a Drosophila simulans genome
}

\author{
Pierre Nouhaud \\ Institut für Populationsgenetik, Vetmeduni, Vienna, Austria \\ Current address: Organismal \& Evolutionary Biology Research Programme, \\ University of Helsinki, Finland \\ Corresponding author: Pierre Nouhaud, pierr3.nouhaud@gmail.com
}

\begin{abstract}
Long-read sequencing technologies enable high-quality, contiguous genome assemblies. Here we used SMRT sequencing to assemble the genome of a Drosophila simulans strain originating from Madagascar, the ancestral range of the species. We generated $8 \mathrm{~Gb}$ of raw data ( $~ 50 \times$ coverage) with a mean read length of $6,410 \mathrm{bp}$, a NR50 of 9,125 $\mathrm{bp}$ and the longest subread at $49 \mathrm{~kb}$. We benchmarked six different assemblers and merged the best two assemblies from Canu and Falcon. Our final assembly was $127.41 \mathrm{Mb}$ with a $\mathrm{N} 50$ of $5.38 \mathrm{Mb}$ and 305 contigs. We anchored more than $4 \mathrm{Mb}$ of novel sequence to the major chromosome arms, and significantly improved the assembly of peri-centromeric and telomeric regions. Finally, we performed full-length transcript sequencing and used this data in conjunction with short-read RNAseq data to annotate 13,422 genes in the genome, improving the annotation in regions with complex, nested gene structures.
\end{abstract}

Keywords. genome assembly, PacBio, SMRT sequencing, IsoSeq Running title. Long-read assembly of $D$. simulans 


\section{Introduction}

DNA sequencing has experienced three revolutions which have profoundly impacted life sciences (Shendure et al. 2017). Forty years ago, Sanger et al. (1977) pioneered the development of a method which would allow to sequence genomes for the first time, starting with phages and culminating with humans. The cost and labor-intensiveness of this method led to the development of second-generation sequencing (SGS) technologies in the early 2000s. While Sanger sequencing produced reads of 600-1000 $\mathrm{bp}$, SGS platforms would generate massive amounts of small reads (e.g., $125 \mathrm{bp}$ for an Illumina HiSeq 2500) at a fraction of the price. SGS technologies are well-suited for resequencing studies but are limited for de novo genome assembly, since the length of DNA repeats is usually more important than the length of a single read. This issue would lead to assembly errors and fragmented genome assemblies (Alkan et al. 2011). The last revolution to date came from long-read, third-generation sequencing (TGS) technologies, which routinely produce reads of more than $10 \mathrm{~kb}$ (van Dijk et al. 2018). TGS can finally overcome the limitations associated with short reads for de novo genome assembly. However, their high error rate $(>10 \%$ for PacBio's SMRT sequencing) requires a sequence correction step either by using SGS data, or by increasing TGS coverage. Another potential application of TGS with SMRT technology is full-length transcript sequencing. Transcript reconstruction from short reads often misses terminal exons or splice junctions (Steijger et al. 2013) and SMRT sequencing would provide better evidence for alternative splicing while improving the characterization of gene models (van Dijk et al. 2018).

The fruit fly Drosophila simulans has diverged from the model organism $D$. melanogaster 2-8 million years ago (Obbard et al. 2012). The first published D. simulans genome was sequenced at low coverage and was obtained from a mixture of strains (Begun et al. 2007; available on FlyBase: flybase.org). One of these strains (strain w501, from North America) was also sequenced at a deeper coverage ( $\mathrm{Hu}$ et al. 2013). Palmieri et al. (2015) assembled the genome of a strain from Madagascar (strain M252), which represents D. simulans ancestral range (Kopp et al. 2006). All three assemblies were based on SGS and were comparable in terms of completeness. 
Here, we report the sequencing and assembly of the $D$. simulans M252 genome using PacBio SMRT technology. We have also annotated the genome using ab initio predictions, RNAseq and full-length transcript sequencing using PacBio SMRT technology. These high-quality assembly and annotation will represent an important resource for population genomic studies in D. simulans and will help our understanding of genome evolution in Drosophila.

\section{Methods}

\section{DNA extraction $\mathcal{E}$ long-read sequencing}

We sequenced the D. simulans M252 strain provided by D.J. Begun and collected in 1998 by W. Ballard in Madagascar. This strain has been maintained in the lab through full-sib mating for several years and residual heterozygosity is expected to be low. High molecular weight DNA was extracted from a pool of larvae using a DNeasy Blood and Tissue Kit (Qiagen, Valencia, CA). DNA was sheared using a Covaris (Woburn, MA) g-TUBE and a single SMRTbell library was prepared using a DNA Template Prep Kit 1.0 and a DNA/Polymerase Binding Kit P6 v2 (Pacific Biosciences, Menlo Park, CA). This library was sequenced using 14 SMRTcells on PacBio RS II with C4 sequencing chemistry. Adaptors were removed and subreads filtered with SMRTanalysis version 1.4 using default parameters.

\section{De novo assembly}

Third generation sequencing platforms have high error rates $(>10 \%$, Rhoads \& $\mathrm{Au}$ 2015) and a correction step is usually needed to build consensus sequences from raw reads. Most of the assemblers developed for these platforms implement this correction step, which requires substantial long read data coverage (at least 30x, Koren et al. 2017). As our sequencing depth was $\sim 50 \times$ (see Results), we generated assemblies from subreads using different algorithms developed for long read data: HGAP in SMRTanalysis 1.4 (Chin et al. 2013), Falcon 0.3 (Chin et al. 2016), Canu 1.4 (Koren et al. 2017), miniasm 0.2 ( $\mathrm{Li} 2016$, no consensus step) and miniasm with Racon 0.5 for error correction (Vaser et al. 2017). Additionally we ran two hybrid assemblers, Spades 3.10 (Bankevich et al. 2012) and MaSuRCA 3.2.1 (Zimin et al. 2017) using long read data in conjunction with $>250 \times$ Illumina data previously generated from the same strain by 
Palmieri et al. (2015, 200 million 100bp paired-end reads sequenced on Illumina HiSeq2000). Apart from Falcon, which by default produced < $60 \mathrm{Mb}$ assemblies (see File S1 for the final, optimized parameter file), all assemblers were run with default parameters, assuming when required an estimated genome size of $162 \mathrm{Mb}$ (Bosco et al. 2007).

For each assembly, contigs were aligned to the $D$. melanogaster reference genome release 6.03 using the progressiveMauve algorithm from Mauve 2.4.0 (Darling et al. 2010). Since genome of both species are colinear (apart from a major chromosomal inversion in the $3 \mathrm{R}$ chromosome arm of the D. melanogaster iso-1 reference strain, which was reverse-complemented prior to this analysis), putative errors were detected when contigs aligned on two different $D$. melanogaster chromosome arms. Note that over all assemblies, no contig spanned centromeric regions.

We then selected the two best assemblies based on their total length, contiguity and absence of assembly errors (see Results). For each of them, two rounds of error correction were performed with Quiver in SMRTanalysis 1.4 using raw PacBio reads, and two additional rounds were done with Pilon 1.21 (Walker et al. 2014) using the aforementioned short read data from Palmieri et al. (2015). These two polished assemblies were combined using Quickmerge 0.2 (Chakraborty et al. 2016), and the same error correction pipeline was applied to the merged assembly.

Contigs from the merged assembly were anchored on the D. melanogaster genome r6 to create chromosome-level scaffolds using the nucmer module from the MUMmer 3.23 package (Delcher et al. 2002; Kurtz et al. 2004). Contigs were arranged into scaffolds using the show-tiling module from the MUMmer package following Nolte et al. (2013). The resulting assembly is hereafter referred to as D. simulans M252 genome version $2(\mathrm{v} 2)$.

\section{Quality assessment}

Assembly quality was assessed using an independent $D$. simulans sample derived from a pool of 202 isofemale lines collected in Florida and containing 200 million reads (population AP1 from Nouhaud et al. 2016, sequenced on Illumina HiSeq2000 with 100-bp paired-end reads, mean insert size: 374 bp). Reads were trimmed using the MottQualityTrimmer in ReadTools (Gómez-Sánchez \& Schlötterer 2018, minimum 
quality: 20, minimum read length: $50 \mathrm{bp}$ ). Mapping was done successively against the D. simulans M252 genome version 1.1 (Palmieri et al. 2015), and against the version 2, using Bowtie2 2.2.6 (Langmead \& Salzberg 2012, parameters --phred33 --end-to-end X 1500) with DistMap (Pandey \& Schlötterer 2013). For each resulting BAM file, we recovered the average percentage of nonproper pairs for non-overlapping, $10 \mathrm{~kb}$ sliding windows using the broken-pairs.pl script from Nolte et al. (2013). Nonproper pairs were defined as pairs were one of the mates (i) did not map, (ii) mapped to a different chromosome, (iii) mapped to the same strand as the other mate, or (iv) when distance between mates was greater than expected.

Genome completeness was assessed through BUSCO gene set analysis 3.0.2 (Simão et al. 2015; Waterhouse et al. 2018) using the Diptera gene set (2,799 orthologs).

\section{RNA extraction, long-read sequencing $\mathcal{E}$ IsoSeq pipeline}

Flies from the M252 strain were maintained under a fluctuating thermal regime (12 hours under light at $28^{\circ} \mathrm{C}, 12$ hours under dark at $18^{\circ} \mathrm{C}$ ) with density control $(400$ eggs per $300 \mathrm{ml}$ bottle, containing $70 \mathrm{ml}$ of standard Drosophila medium). After three generations, 50 males were frozen in liquid nitrogen in the middle of each temperature window. High quality RNA was extracted from each pool of male whole bodies using a RNeasy Plus Universal Mini Kit (Qiagen, Valencia, CA) and one SMRTbell library was built per sample (collected at 18 and $28^{\circ} \mathrm{C}$, hence two libraries in total) following the Iso-Seq template preparation procedure from PacBio. Each library was sequenced using a single SMRTcell on PacBio Sequel with V2 chemistry at the VBCF NGS Unit (www.vbcf.ac.at). Raw data was processed using the IsoSeq pipeline (Gordon et al. 2015, available at https://github.com/PacificBiosciences/IsoSeq_SA3nUP) following PacBio's guidelines. Briefly, the different steps are:

1. Identification of full-length (FL) reads for which all 5' -primer, polyA tail and $3^{\prime}$ primer have been sequenced;

2. Clustering of FL reads at the isoform level;

3. Alignment of non-FL reads to the isoform clusters;

4. Error correction using both FL and non-FL reads with the Arrow model. This de novo pipeline outputs FASTQ files containing two sets of error-corrected, fulllength isoforms: the high-quality set contains isoforms supported by at least 2 FL reads 
with an accuracy of at least $99 \%$, while isoforms from the low-quality set display an accuracy $<99 \%$ (reasons could be insufficient coverage or rare transcripts). The pipeline was run first by pooling both libraries (giving a high-confidence set of transcripts for annotation, see below) and then for each library independently.

\section{Annotation}

Repeats in the v2 genome were masked with RepeatMasker (http://www.repeatmasker.org/) after initial training based on D. melanogaster. We then used Maker 2.31 (Cantarel et al. 2008) to annotate the genome by first incorporating in silico gene models detected by Augustus 3.3 (Stanke \& Morgenstern 2005), a de novo D. simulans transcriptome built with RNA-Seq data from Palmieri et al. (2015, single library combining multiple developmental stages and sequenced on Illumina HiSeq2000 with 100-bp paired-end reads, see initial publication for details) using Trinity r2014-07-17 (Haas et al. 2013) and all RefSeq protein sequences available for D. simulans on NCBI $(n=295,428$, accessed on 31/10/2017), along with protein sequences from D. melanogaster r6.15 obtained from FlyBase (Gramates et al. 2017). The software was allowed to take extra steps to identify alternate splice variants.

Visual inspection of the output and comparison to the D. melanogaster annotation showed many gene fusion events and incorrect reconstruction of nested gene structures (see Results and Fig. 2). No significant improvement was detected after (i) decreasing the physical distance used to extend evidence clusters sent to gene predictors (pred_flank parameter, set to $100 \mathrm{bp}$ instead of default $200 \mathrm{bp}$ ) and (ii) limiting the use of RNA-Seq data during the annotation to reduce gene fusion events (correct_est_fusion parameter disabled), as recommended in the Maker documentation.

To solve this issue, we included the IsoSeq data in the annotation procedure. The IsoSeq pipeline was ran simultaneously on the two combined libraries. The two resulting FASTQ files containing low and high quality sets of isoforms were pooled and aligned against the v2 genome using gmap r2017-10-12 (Wu \& Nacu 2010) and only alignments with $>90 \%$ identity were kept. The resulting BAM file was reverted as a FASTA file and replaced the Trinity short-read-based transcriptome assembly in the Maker pipeline. While IsoSeq data was acquired from adult males only, short-read 
RNA-Seq data was generated from a pool of developmental stages and sexes (see Palmieri et al. 2015 for details), making it useful for isoform detection. We included this data in our annotation procedure as an additional file by mapping it against the v2 genome using gsnap r2017-10-12 (Wu \& Nacu 2010) with default parameters. The resulting BAM file was filtered for proper pairs using SAMtools 1.5 ( $\mathrm{Li}$ et al. 2009). Since transcript reconstruction can be confounded by high coverage (Palmieri et al. 2012), a subset of randomly sampled 50M read pairs was used in Cufflinks 2.2.1 (Trapnell et al. 2010) with default parameters. The resulting transcript file was converted from gtf to gff3 format and used as est_gff input in Maker.

To sum up, the modified annotation pipeline contained two differences compared to the initial one: (i) using IsoSeq data instead of Trinity assembly, and (ii) adding the Cufflinks transcriptome assembly from short read data as additional input file. All other parameters, options and input files (Augustus de novo gene prediction, protein sequence data) were similar between the two pipelines.

\section{Data availability}

All PacBio data (DNA sequencing and IsoSeq) and the D. simulans M252 v2 genome assembly are available from the European Nucleotide Archive (ENA project ID PRJEB28741). The output of the IsoSeq pipeline (fastq files) and annotation tracks are available from Dryad.

\section{Results \& Discussion}

\section{DNA long read sequencing}

We sequenced DNA of an inbred D. simulans line from Madagascar using 14 SMRTcells on a PacBio RS II. After filtering and adapter removal, this led to $8 \mathrm{~Gb}$ of raw data spanning 745,625 subreads with a mean read length of $6,410 \mathrm{bp}$, the longest subread reached 48,980 bp and the NR50 was 9,125 bp (NR50 is the read length such that $50 \%$ of the total sequence is contained within reads of at least this length). Mean coverage of the v1 reference genome (Palmieri et al. 2015) was $\sim 50 \times$ after mapping subreads with BWA mem (Li 2013, with option -x pacbio).

\section{Benchmarking of assembly algorithms}


We ran seven different algorithms to assemble the genome (Table 1). While all resulting assemblies were fairly similar size-wise (124 to $135 \mathrm{Mb}$ ), they differed in terms of fragmentation (number of contigs and N50 respectively ranging from 311 to 107,481 and from $610 \mathrm{~kb}$ to $5.37 \mathrm{Mb}$ ). Alignment of assemblies to the D. melanogaster reference genome r6.03 revealed all algorithms but Falcon, Canu and SPAdes produced errors with this data set. Since HGAP was developed for bacterial genome assembly, it is not optimized for eukaryote genomes. miniasm do not perform any consensus step and as such may erroneously assemble reads, and the Racon polishing step is not sufficient to uncover these assembly errors. The MaSuRCA assembly contained an assembly error on the longest contig (hence its size, Table 1) and overall the output of hybrid assemblers was much more fragmented than for PacBio-only assemblers, since many Illumina-only contigs inflated the assembly. In line with a recent benchmark study (Jayakumar \& Sakakibara 2017), Falcon and Canu overall provided the best assemblies, which were both polished using long- and short-read data (Quiver and Pilon, two iterations each). Polished assemblies were merged using Quickmerge, using the Canu assembly as query. This merged assembly encompassed 127.41 Mb on 305 contigs, with a N50 of $5.38 \mathrm{Mb}$. Interestingly, the merged assembly was only marginally better in terms of contiguity and total size, while bigger improvement is usually observed with this tool (e.g., Chakraborty et al. 2016; Mahajan et al. 2018). This would be expected if both assemblies were already fairly similar, as suggested by their statistics.

Table 1. Assembly statistics obtained for $D$. simulans using seven different algorithms with $\sim 50 \times$ PacBio data. Hybrid assemblers were run using this data in conjunction with $>250 \times$ Illumina paired-end data from Palmieri et al. (2015). \#: Assembly errors are defined as contigs aligning on two different $D$. melanogaster chromosome arms.

\begin{tabular}{lcccccc} 
Assembler & $\begin{array}{c}\text { Assembler } \\
\text { type }\end{array}$ & $\begin{array}{c}\text { Assembly } \\
\text { size } \mathbf{( M b )}\end{array}$ & $\begin{array}{c}\text { Number } \\
\text { of contigs }\end{array}$ & $\begin{array}{c}\text { N50 } \\
\mathbf{( M b})\end{array}$ & $\begin{array}{c}\text { Longest } \\
\text { contig (Mb) }\end{array}$ & $\begin{array}{c}\text { Assembly } \\
\text { errors\# }\end{array}$ \\
\hline HGAP & & 135.30 & 1508 & 4.49 & 11.20 & Yes \\
Falcon & PacBio data & 124.35 & 370 & 5.37 & 16.52 & No \\
Canu & only & 127.35 & 311 & 5.23 & 16.55 & No \\
miniasm & & 132.39 & 521 & 1.82 & 6.40 & Yes \\
miniasm + Racon & & 129.03 & 521 & 1.78 & 6.24 & Yes \\
MaSuRCA & Hybrid & 130.72 & 857 & 4.02 & 25.90 & Yes \\
SPAdes & & 131.63 & 107,481 & 0.61 & 3.10 & No \\
\hline
\end{tabular}


Small indels are the most frequent sequencing errors associated to the PacBio technology (Rhoads \& Au 2015). Polishing the merged assembly with both PacBio (twice, using Quiver) and Illumina (twice, using Pilon) reads greatly reduced these errors (number of indels per $100 \mathrm{~kb}$ : unpolished $=136$; polished $=5.03$; 27-fold reduction). A significant fraction of mismatches was still present after polishing (number of mismatches per $100 \mathrm{~kb}$ : unpolished $=25.4$; polished $=19.1$ ) However, these values were obtained by comparing both assembly versions assuming the sequence of the v1 assembly was error-free, which is highly unlikely.

Table 2. Assembly size comparison of the two $D$. simulans genome versions (v1: Illuminabased, v2: PacBio-based) after anchoring of the PacBio contigs on the D. melanogaster reference genome r6. $\Delta$ size: size difference between v1 and v2 assemblies.

\begin{tabular}{lccccc}
\hline \multirow{2}{*}{ Chromosome } & \multicolumn{2}{c}{ Size $\mathbf{( M b )}$} & \multicolumn{2}{c}{$\boldsymbol{\Delta}$ size } & \multicolumn{2}{c}{ Number of contigs } \\
& $\mathbf{v} \mathbf{1}$ & $\mathbf{v} \mathbf{2}$ & $\mathbf{( M b )}$ & $\mathbf{v 1}$ & $\mathbf{v 2}$ \\
\hline $\mathrm{X}$ & 20.62 & 21.52 & 0.90 & 412 & 26 \\
2L & 21.09 & 21.87 & 0.78 & 491 & 8 \\
2R & 18.98 & 19.92 & 0.94 & 307 & 16 \\
3L & 22.25 & 22.88 & 0.63 & 229 & 10 \\
3R & 26.97 & 27.78 & 0.81 & 265 & 15 \\
4 & 1.10 & 1.12 & 0.02 & 42 & 2 \\
$\quad$ Sum & 111.01 & 115.08 & 4.07 & & \\
Unassembled & 10.19 & 12.23 & 2.05 & 2941 & 228 \\
$\quad$ Overall sum & 121.20 & 127.32 & 6.12 & & \\
\hline
\end{tabular}

\section{Anchoring on the D. melanogaster reference genome}

To achieve a chromosome-level assembly, polished contigs were anchored on the $D$. melanogaster r6 reference using nucmer. After alignment, the number of contigs per chromosome varied from two (chromosome 4) to 26 (X chromosome, Table 2). Compared to the v1 assembly, our PacBio-based v2 assembly contained more than six $\mathrm{Mb}$ of novel sequence, among which four were located on major chromosome arms. Overall our v2 assembly was $127.32 \mathrm{Mb}$, with almost five percent increase in size compared to the v1. Most of this novel sequence located within repeat-rich, telomeric or peri-centromeric regions (Figure S1), for which assembly from short-read data is challenging (van Dijk et al. 2018).

While the SMRTbell library was built using a pool of larvae (i.e., both sexes), we did not manage to assemble the $\mathrm{Y}$ chromosome, which is highly repetitive (Mahajan et al. 2018). The relative low amount of Y-linked sequences expected in our library 
compared to autosomes (hence, low coverage) could have been a major impediment to take on this task.
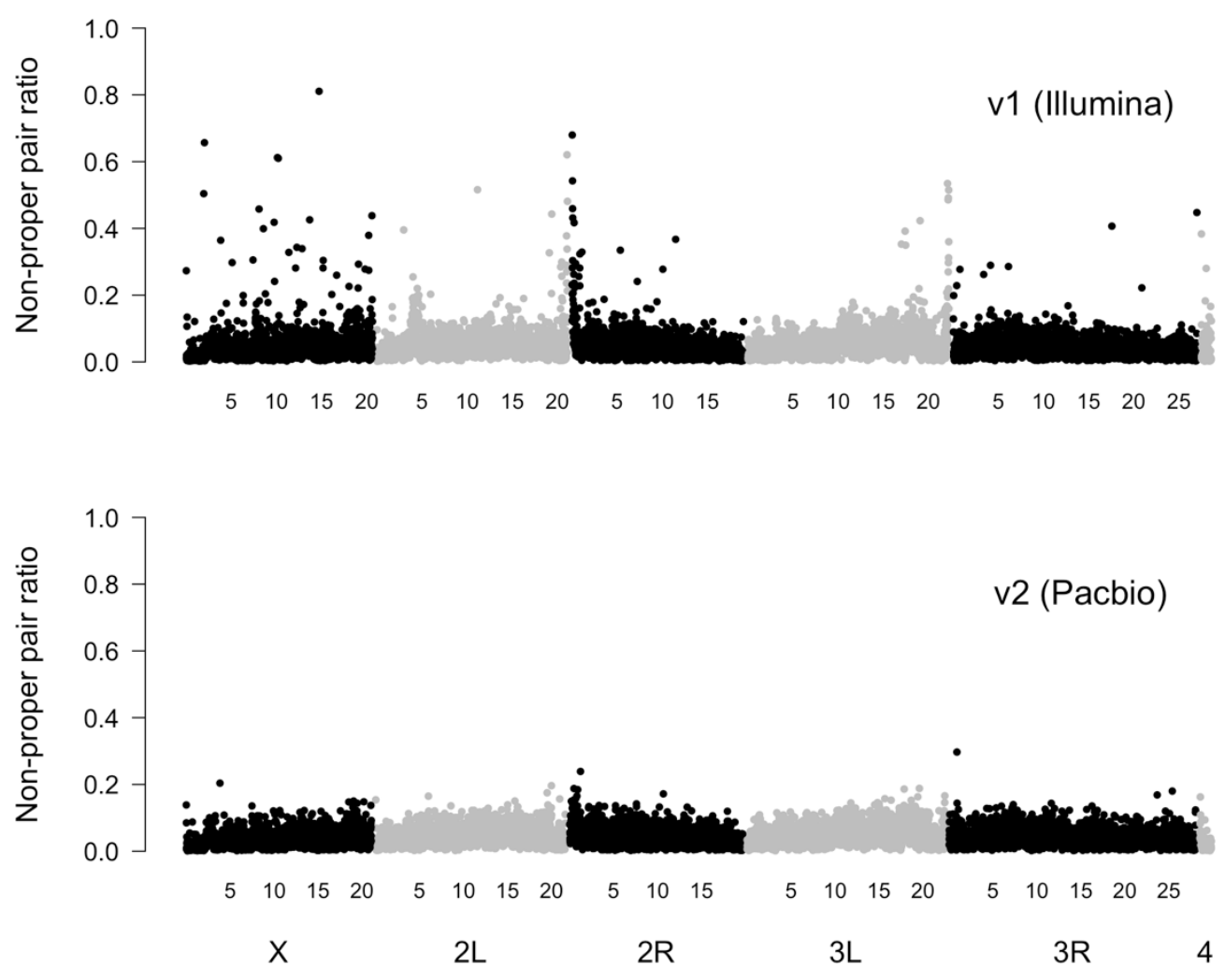

Figure 1. Improved contiguity of the PacBio assembly. The ratio of non-proper Illumina read pairs was recovered in non-sliding $10 \mathrm{~kb}$ windows along the genome for the same dataset aligned against the v1, Illumina-based assembly (Palmieri et al. 2015, upper panel) and the v2, PacBio-based assembly (lower panel). Coordinates on the $\mathrm{x}$-axis are in $\mathrm{Mb}$.

\section{Assembly quality}

Nonproper read pairs could reveal the presence of misassemblies in the reference genome. We recovered the ratio of nonproper pairs in non-overlapping, $10 \mathrm{~kb}$ windows using an independent pool of isofemale lines collected in Florida which were aligned on both the v1 and v2 reference genomes. For all major chromosome arms, the mean ratio was smaller for the v2 assembly (see Table S1), and especially for the $X$ chromosome (v1: 4.57\%; v2: 3.59\%). We plotted the ratio of nonproper pairs per window to obtain a genome-wide distribution of misassemblies for both versions (Figure 1). Most of the assembly errors found in the v1 along the $\mathrm{X}$ chromosome and in peri-centromeric regions were actually corrected in the v2, since these loci were assembled with less, longer contigs (Table 2). However, the v1 assembly was fairly good outside of these regions and less improvement was observed in the v2, apart 
from a region between 4 and $5 \mathrm{Mb}$ on chromosome 2L. Since the v1 assembly was comparable to both the FlyBase r.14 (Begun et al. 2007) and the $\mathrm{Hu}$ et al. (Hu et al. 2013) assemblies in terms of quality (Palmieri et al. 2015), this suggests the v2 also outperforms these assemblies in terms of contiguity and assembly correctness.

We assessed assembly completeness through the BUSCO analysis, using the Diptera gene set $(n=2799$ orthologs). Our v2 assembly contained $98.7 \%$ of the orthologs among which $98.2 \%$ were complete and in single-copy, while $0.6 \%$ were detected as fragmented. Only $0.7 \%$ of the orthologs were not found in our assembly. We ran the BUSCO analysis on the D. melanogaster r6.17 reference and completeness of both genomes was comparable (Table 3).

Table 3. Gene content assessment for $D$. simulans v2 and D. melanogaster r6.17 assemblies using the BUSCO (Benchmarking Universal Single-Copy Ortholog) Diptera gene set $(n=$ 2799 orthologs).

\begin{tabular}{lcc}
\hline Category & D. simulans v2 & D. melanogaster $\mathbf{r 6 . 1 7}$ \\
\hline Complete Single-Copy (\%) & 98.2 & 98.2 \\
Duplicated (\%) & 0.5 & 0.5 \\
Fragmented (\%) & 0.6 & 0.8 \\
Missing (\%) & 0.7 & 0.5 \\
\hline
\end{tabular}

\section{RNA long read sequencing}

We extracted RNA from two pools of D. simulans males from the M252 strain and sequenced two SMRT libraries using PacBio. Each cDNA library produced $\sim 5 \mathrm{~Gb}$ of raw data and 245,000 subreads, with a mean read length of 1,916 bp. The IsoSeq pipeline was run on the two merged libraries to produce 28,250 high and 115,918 low quality isoforms with a mean length of $2,484 \mathrm{bp}$. The two sets of isoforms were pooled and mapped against the v2 reference genome and isoforms aligning with at least $90 \%$ identity were kept for the annotation step.

\section{Annotation}

We used the Maker pipeline to annotate the genome by combining ab initio predictions from Augustus, RNAseq data from Palmieri et al. (2015) and protein sequence data from D. melanogaster and D. simulans. Visual comparison with the D. melanogaster r6.17 annotation in FlyBase revealed two issues: nested gene structures were misidentified 
(Figure 2), and gene fusion events occurred when UTRs overlapped. Following Maker's guidelines (see Methods) did not solve these issues. Inclusion of the IsoSeq data in the annotation pipeline allowed us to reconstruct correctly such gene structures while limiting gene fusion events (Figure 2). This improved pipeline recovered 13,422 genes and 18,301 transcripts (mean number of isoforms per gene: 1.36). The mean gene length was 5,335 bp and the mean number of exons per gene was 2.98 , with a mean exon length of $492 \mathrm{bp}$ and a mean intron length of $967 \mathrm{bp}$. Overall, genes accounted for $56.4 \%$ of the genome. These numbers are in line with previous observations made for D. simulans (Begun et al. 2007; Hu et al. 2013; Palmieri et al. 2015).

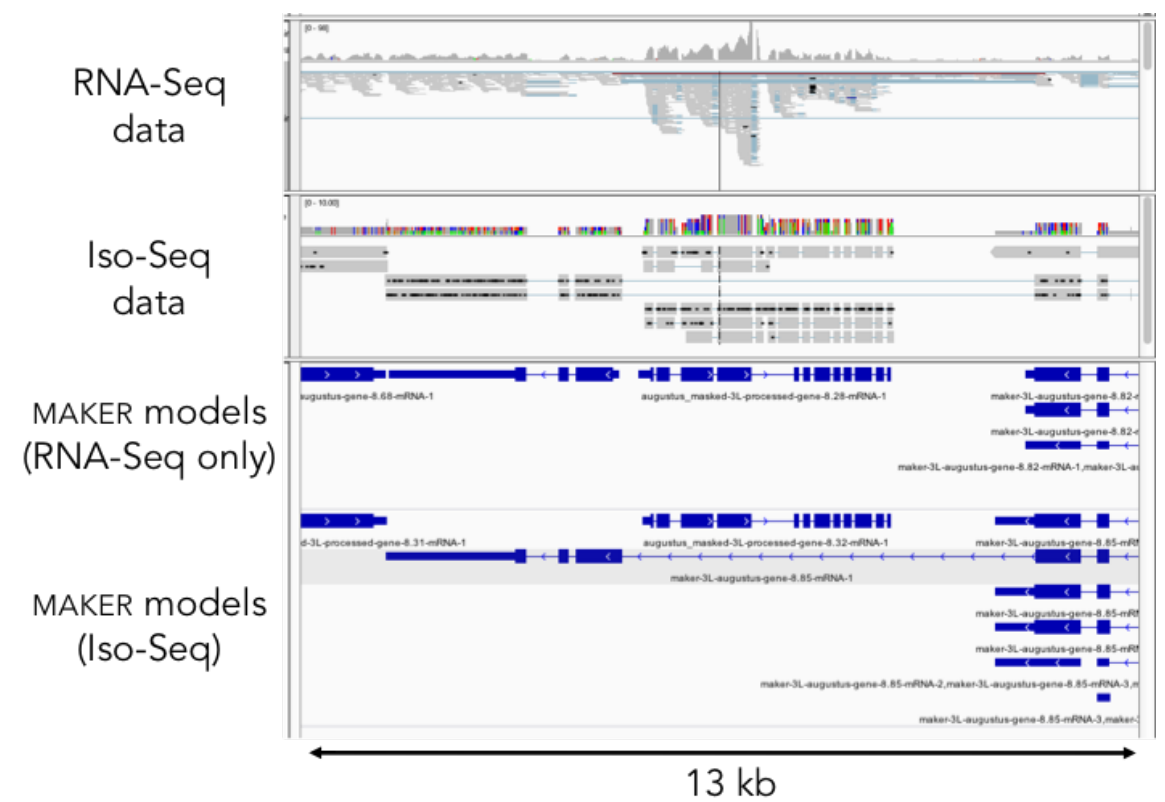

Figure 2. IsoSeq data helps automated genome annotation. Maker models (third panel from top, in IGV) built from RNA-Seq data (top panel) cannot resolve the nested gene structure, while Maker models (bottom panel) built from IsoSeq data (second panel from top) can.

Maker uses the Annotation Edit Distance (AED) to measure the agreement between ab initio predictions and empirical evidence for each gene model (0 indicates perfect agreement whereas 1 indicates no support of empirical data). Our gene models had a mean AED of 0.18 (median: 0.13 ) and $98 \%$ of them had a distance smaller than 0.5. Finally, we could validate $90 \%$ of our gene models by best reciprocal BLAST against D. melanogaster r6.17 transcripts. These results suggest most of our annotations are of high quality. Our study demonstrates that PacBio sequencing of full-length transcripts associated with the IsoSeq pipeline represent a sound approach to build high-quality 
annotations. Depending on the size of the transcriptome, and with the decreasing costs of PacBio sequencing, this could represent a promising strategy also in non-model organisms.

\section{Conclusion}

This work provides the first complete long-read-based assembly of a $D$. simulans genome and adds to the platinium-grade assemblies available for Drosophila (Chakraborty et al. 2018; Mahajan et al. 2018; Miller et al. 2018). With a high contiguity level and a gene content completeness comparable to the D. melanogaster genome, this will provide a useful resource for genomic studies. The better assembly of pericentromeric and telomeric regions could also allow to investigate the evolution of transposable elements at an unprecedented scale. Finally, including IsoSeq data in the annotation improved characterization of complex gene structures and should thus provide a better resolution for future RNAseq studies in $D$. simulans.

\section{Acknowledgments}

I wish to acknowledge Viola Nolte for library preparation, Lukas Endler for assisting with SMRTanalysis installation, Heinz Ekker from the VBCF for running the IsoSeq pipeline and Christian Schlöterrer and colleagues from the Institut für Populationsgenetik for constructive feedback. This research was supported by the European Research Council grant "Archadapt" awarded to Christian Schlöterrer and a Bright Spark grant of the vetmeduni Vienna awarded to PN. The funders had no role in study design, data collection and analysis, decision to publish, or preparation of the manuscript. I declare no conflict of interest.

\section{Literature cited}

Alkan C, Coe BP, Eichler EE. 2011. Genome structural variation discovery and genotyping. Nat. Rev. Genet. 12:363-376. doi: 10.1038/nrg2958.

Bankevich A et al. 2012. SPAdes: a new genome assembly algorithm and its applications to single-cell sequencing. J. Comput. Biol. 19:455-77. doi: 10.1089/cmb.2012.0021.

Begun DJ et al. 2007. Population genomics: Whole-genome analysis of polymorphism and divergence in Drosophila simulans. PLoS Biol. 5:e310. doi: 10.1371/journal.pbio.0050310.

Bosco G, Campbell P, Leiva-Neto JT, Markow TA. 2007. Analysis of Drosophila species genome size and satellite DNA content reveals significant differences among strains as well as between species. Genetics. 177:1277-90. doi: 10.1534/genetics.107.075069.

Cantarel BL et al. 2008. MAKER: an easy-to-use annotation pipeline designed for emerging model 
organism genomes. Genome Res. 18:188-96. doi: 10.1101/gr.6743907.

Chakraborty $\mathrm{M}$ et al. 2018. Hidden genetic variation shapes the structure of functional elements in Drosophila. Nat. Genet. 50:20-25. doi: 10.1038/s41588-017-0010-y.

Chakraborty M, Baldwin-Brown JG, Long AD, Emerson JJ. 2016. Contiguous and accurate de novo assembly of metazoan genomes with modest long read coverage. Nucleic Acids Res. 44:1-12. doi: $10.1093 /$ nar/gkw654.

Chin C-S et al. 2013. Nonhybrid, finished microbial genome assemblies from long-read SMRT sequencing data. Nat. Methods. 10:563-569. doi: 10.1038/nmeth.2474.

Chin C-S et al. 2016. Phased diploid genome assembly with single-molecule real-time sequencing. Nat. Methods. 13:1050-1054. doi: 10.1038/nmeth. 4035.

Darling AE, Mau B, Perna NT. 2010. progressiveMauve: Multiple Genome Alignment with Gene Gain, Loss and Rearrangement Stajich, JE, editor. PLoS One. 5:e11147. doi: 10.1371/journal.pone.0011147.

Delcher AL, Phillippy A, Carlton J, Salzberg SL. 2002. Fast algorithms for large-scale genome alignment and comparison. Nucleic Acids Res. 30:2478-2483. doi: 10.1093/nar/30.11.2478.

van Dijk EL, Jaszczyszyn Y, Naquin D, Thermes C. 2018. The Third Revolution in Sequencing Technology. Trends Genet. 34:666-681. doi: 10.1016/j.tig.2018.05.008.

Gómez-Sánchez D, Schlötterer C. 2018. ReadTools : A universal toolkit for handling sequence data from different sequencing platforms. Mol. Ecol. Resour. 18:676-680. doi: 10.1111/1755-0998.12741.

Gordon SP et al. 2015. Widespread Polycistronic Transcripts in Fungi Revealed by Single-Molecule mRNA Sequencing Zheng, D, editor. PLoS One. 10:e0132628. doi: 10.1371/journal.pone.0132628.

Gramates LS et al. 2017. FlyBase at 25: looking to the future. Nucleic Acids Res. 45:D663-D671. doi: $10.1093 /$ nar/gkw1016.

Haas BJ et al. 2013. De novo transcript sequence reconstruction from RNA-seq using the Trinity platform for reference generation and analysis. Nat. Protoc. 8:1494-1512. doi: 10.1038/nprot.2013.084.

Hu TT, Eisen MB, Thornton KR, Andolfatto P. 2013. A second-generation assembly of the Drosophila simulans genome provides new insights into patterns of lineage-specific divergence. Genome Res. 23:89-98. doi: 10.1101/gr.141689.112.

Jayakumar V, Sakakibara Y. 2017. Comprehensive evaluation of non-hybrid genome assembly tools for third-generation PacBio long-read sequence data. Brief. Bioinform. doi: 10.1093/bib/bbx147.

Kopp A, Frank A, Fu J. 2006. Historical biogeography of Drosophila simulans based on Ychromosomal sequences. Mol. Phylogenet. Evol. 38:355-362. doi: 10.1016/J.YMPEV.2005.06.006.

Koren $S$ et al. 2017. Canu: scalable and accurate long-read assembly via adaptive k-mer weighting and repeat separation. Genome Res. 27:722-736. doi: 10.1101/gr.215087.116.

Kurtz S et al. 2004. Versatile and open software for comparing large genomes. Genome Biol. 5:R12. doi: 10.1186/gb-2004-5-2-r12.

Langmead B, Salzberg SL. 2012. Fast gapped-read alignment with Bowtie 2. Nat. Methods. 9:357-359. doi: $10.1038 /$ nmeth.1923.

Li H. 2013. Aligning sequence reads, clone sequences and assembly contigs with BWA-MEM. http://arxiv.org/abs/1303.3997 (Accessed February 12, 2018).

Li H. 2016. Minimap and miniasm: Fast mapping and de novo assembly for noisy long sequences. Bioinformatics. 32:2103-2110. doi: 10.1093/bioinformatics/btw152.

$\mathrm{Li} \mathrm{H}$ et al. 2009. The Sequence Alignment/Map format and SAMtools. Bioinformatics. 25:2078-2079. doi: $10.1093 /$ bioinformatics/btp352.

Mahajan S, Wei KH-C, Nalley MJ, Gibilisco L, Bachtrog D. 2018. De novo assembly of a young Drosophila $Y$ chromosome using single-molecule sequencing and chromatin conformation capture. PLOS Biol. 16:e2006348. doi: 10.1371/journal.pbio.2006348.

Miller DE, Staber C, Zeitlinger J, Hawley RS. 2018. High-quality genome assemblies of 15 Drosophila species generated using Nanopore sequencing. bioRxiv. 267393. doi: 10.1101/267393.

Nolte V, Pandey RV, Kofler R, Schlötterer C. 2013. Genome-wide patterns of natural variation reveal strong selective sweeps and ongoing genomic conflict in Drosophila mauritiana. Genome Res. 23:99110. doi: 10.1101/gr.139873.112.

Nouhaud P, Tobler R, Nolte V, Schlötterer C. 2016. Ancestral population reconstitution from isofemale lines as a tool for experimental evolution. Ecol. Evol. 6. doi: 10.1002/ece3.2402.

Obbard DJ et al. 2012. Estimating Divergence Dates and Substitution Rates in the Drosophila Phylogeny. Mol. Biol. Evol. 29:3459-3473. doi: 10.1093/molbev/mss150.

Palmieri N, Nolte V, Chen J, Schlötterer C. 2015. Genome assembly and annotation of a Drosophila simulans strain from Madagascar. Mol. Ecol. Resour. 15:372-381. doi: 10.1111/1755-0998.12297. 
bioRxiv preprint doi: https://doi.org/10.1101/425710; this version posted September 24, 2018. The copyright holder for this preprint (which was not certified by peer review) is the author/funder, who has granted bioRxiv a license to display the preprint in perpetuity. It is made available under aCC-BY-NC-ND 4.0 International license.

Palmieri N, Nolte V, Suvorov A, Kosiol C, Schlötterer C. 2012. Evaluation of Different Reference Based Annotation Strategies Using RNA-Seq - A Case Study in Drososphila pseudoobscura Zhu, D, editor. PLoS One. 7:e46415. doi: 10.1371/journal.pone.0046415.

Pandey RV, Schlötterer C. 2013. DistMap: A Toolkit for Distributed Short Read Mapping on a Hadoop Cluster Yan, W, editor. PLoS One. 8:e72614. doi: 10.1371/journal.pone.0072614.

Rhoads A, Au KF. 2015. PacBio Sequencing and Its Applications. Genomics, Proteomics Bioinforma. 13:278-289. doi: 10.1016/j.gpb.2015.08.002.

Sanger F, Nicklen S, Coulson AR. 1977. DNA sequencing with chain-terminating inhibitors. Proc. Natl. Acad. Sci. U. S. A. 74:5463-7. http://www.ncbi.nlm.nih.gov/pubmed/271968 (Accessed August 31, 2018).

Shendure J et al. 2017. DNA sequencing at 40: past, present and future. Nature. 550:345-353. doi: 10.1038 /nature24286.

Simão FA, Waterhouse RM, Ioannidis P, Kriventseva E V., Zdobnov EM. 2015. BUSCO: assessing genome assembly and annotation completeness with single-copy orthologs. Bioinformatics. 31:32103212. doi: 10.1093/bioinformatics/btv351.

Stanke M, Morgenstern B. 2005. AUGUSTUS: a web server for gene prediction in eukaryotes that allows user-defined constraints. Nucleic Acids Res. 33:W465-W467. doi: 10.1093/nar/gki458.

Steijger T et al. 2013. Assessment of transcript reconstruction methods for RNA-seq. Nat. Methods. 10:1177-1184. doi: 10.1038/nmeth.2714.

Trapnell C et al. 2010. Transcript assembly and quantification by RNA-Seq reveals unannotated transcripts and isoform switching during cell differentiation. Nat. Biotechnol. 28:511-515. doi: $10.1038 /$ nbt.1621.

Vaser R, Sović I, Nagarajan N, Šikić M. 2017. Fast and accurate de novo genome assembly from long uncorrected reads. Genome Res. 27:737-746. doi: 10.1101/gr.214270.116.

Walker BJ et al. 2014. Pilon: An Integrated Tool for Comprehensive Microbial Variant Detection and Genome Assembly Improvement Wang, J, editor. PLoS One. 9:e112963. doi: 10.1371/journal.pone.0112963.

Waterhouse RM et al. 2018. BUSCO Applications from Quality Assessments to Gene Prediction and Phylogenomics. Mol. Biol. Evol. 35:543-548. doi: 10.1093/molbev/msx319.

Wu TD, Nacu S. 2010. Fast and SNP-tolerant detection of complex variants and splicing in short reads. Bioinformatics. 26:873-881. doi: 10.1093/bioinformatics/btq057.

Zimin A V et al. 2017. Hybrid assembly of the large and highly repetitive genome of Aegilops tauschii, a progenitor of bread wheat, with the MaSuRCA mega-reads algorithm. Genome Res. 27:787-792. doi: 10.1101/gr.213405.116. 


\section{Supplementary data}

Table S1. Mean nonproper pair ratio for the different major chromosome arms obtained from the same data set after mapping to both reference genomes (v1: Illumina-based; v2: PacBio-based).

\begin{tabular}{lcc}
\hline \multirow{2}{*}{ Chromosome arm } & \multicolumn{2}{c}{ Mean nonproper pair ratio } \\
& v1 & v2 \\
\hline X & 0.04566 & 0.03592 \\
2L & 0.05394 & 0.04702 \\
2R & 0.04853 & 0.04434 \\
3L & 0.05387 & 0.0495 \\
3R & 0.04243 & 0.04121 \\
4 & 0.03831 & 0.02163 \\
\hline
\end{tabular}

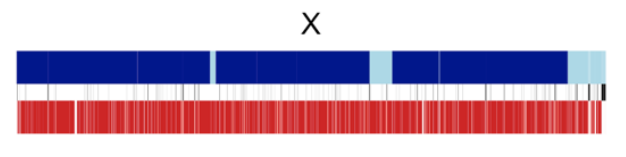

$2 \mathrm{~L}$

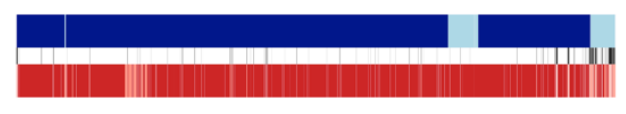

$3 \mathrm{~L}$

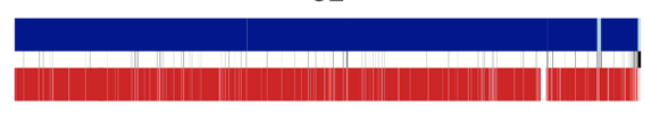

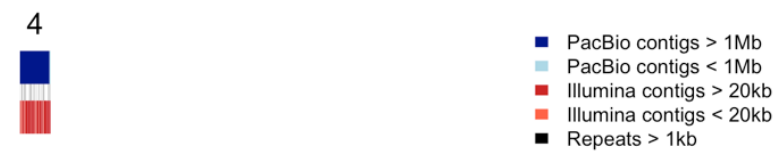

$2 \mathrm{R}$

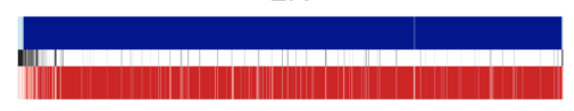

$3 R$

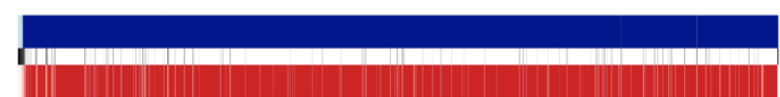

Figure S1. Mapping of PacBio (blue) and Illumina (red) contigs on major chromosomes of the v2 reference genome. Repeats $(>1 \mathrm{~kb})$ were identified by RepeatMasker and are indicated in black. Illumina contigs are from Palmieri et al. (2015). 
bioRxiv preprint doi: https://doi.org/10.1101/425710; this version posted September 24,2018 . The copyright holder for this preprint (which was not certified by peer review) is the author/funder, who has granted bioRxiv a license to display the preprint in perpetuity. It is made available under aCC-BY-NC-ND 4.0 International license.

File S1. Optimized config file used for the Falcon assembler.

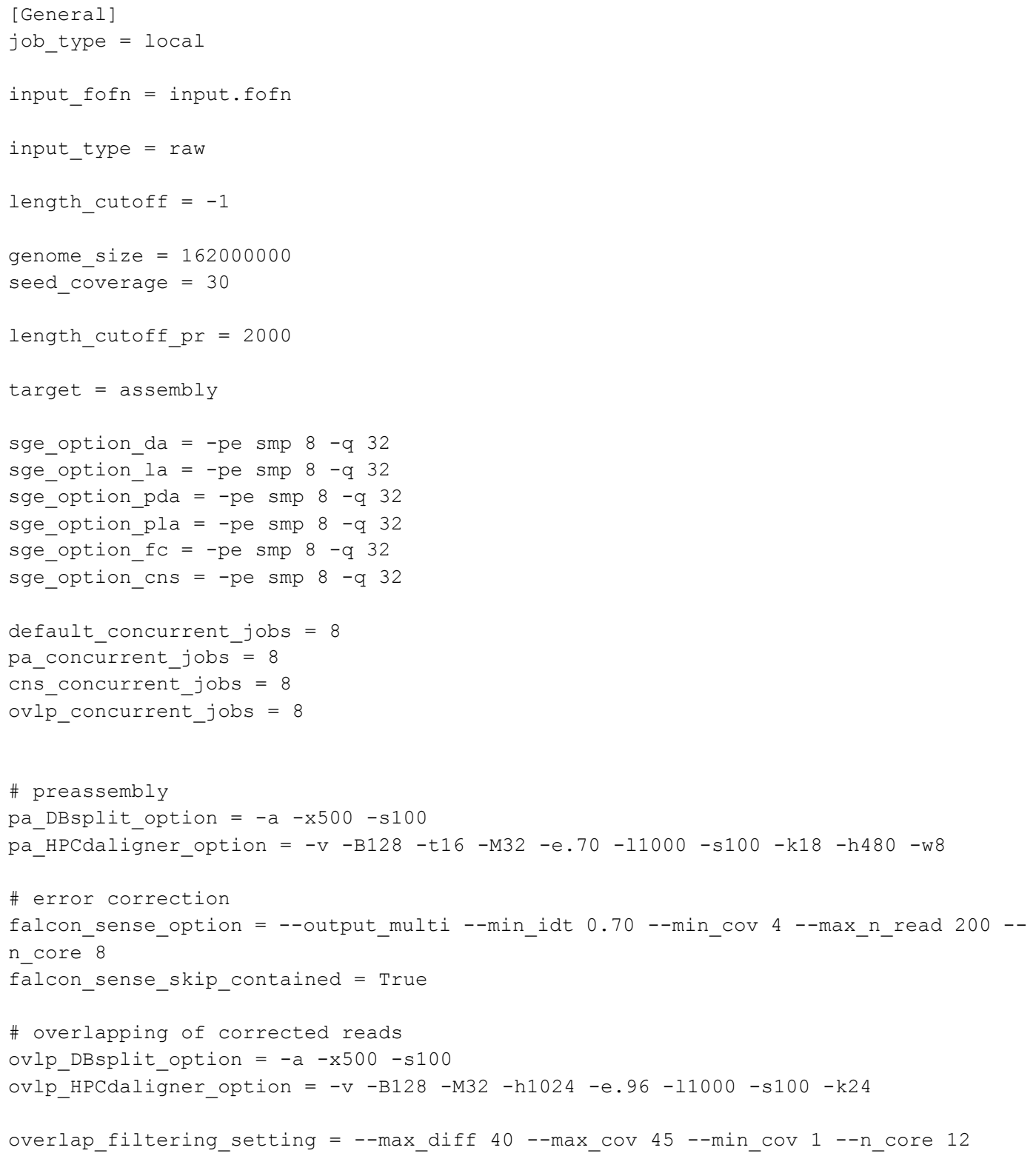

